\title{
RADIO SOURCES AND THEIR ENVIRONMENT (DISCUSSION)
}

Discussion of the paper presented by OWEN (p. 305)

Macchetto: In the cluster merger calculations that you showed, it was not obvious where the centre of mass (i.e. the position of the CD galaxy) was, and therefore whether the gas would indeed stream pass the galaxy and create the bent radio-lobes.

Owen: The center of mass in the merger simulations evolves as the dark matter in the two cluster merge. The gas which is not important gravitationally tries to follow the changing potential but is limited by its sound speed hydrodynamically. This leads to the long lasting, large scale flows which look very different than the potential and last for many Gigayears.

Rudnick: With the improved statistics, can you tell whether a galaxy has a greater probability of being a radio source if another galaxy in the cluster already is a radio source?

Owen: In nearby clusters there are too few radio galaxies per cluster to sort this out, but this is an exciting prospect in the more distant, very rich clusters.

Ekers: It is very satisfying to see that the idea of large scale motions of the cluster gas are now almost acceptable. An early and very clean argument for large scale motion of cluster gas was made by Toomre in the 1970's when he noted that the large scale curvature of the head tail radio source NGC1265 in the Perseus cluster could not be caused by any possible galaxy orbit; this leads directly to the need for large scale motion of the cluster gas with velocity $\geq 1000 \mathrm{~km} / \mathrm{sec}$.

Owen: Yes, the shapes of sources like NGC1265 must be due to a combination of their orbital motion and the velocity field of the cluster gas. The nice thing about the cluster-cluster merger model is that it provides a mechanism for causing the gas motions.

Radhakrishnan: What about using iron's other X-ray lines to measure the motions of cluster gas through Doppler shifts?

Owen: This should be an exciting possibility for future x-ray satellites. 
Binette: Supposing the RG wobble within the X-ray gas along the direction of elongation of the $\mathrm{X}$-ray gas, could this be an indication of the ability of the RG to "heat up". the cooling flow gas? This would reduce the mass inflow rate inferred for "cooling flows".

Discussion of the paper presented by $\underline{D A L Y}$ (p. 319)

Laing: What do you think is the physical reason why the beam power is anticorrelated with the time for which the source is on?

Daly: The beam power $L_{j}$, time the AGN is on $t_{*}$, and initial energy available to power the outflow $E_{i}$ are related: $L_{j}=E_{i} / t_{*}$. Here, it is assumed that the initial energy of a given object is a fixed quantity, as would be the case if it were the spin energy of the central object. If the energy extraction rate $L_{j}$ were independent of the initial energy, then we would have $L_{j} \propto t_{*}^{-1}$, so it is not unreasonable to expect the beam power and time to be anticorrelated. In fact, we were motivated by several puzzling observations that could be understood if we wrote $t_{*} \propto L_{j}^{-\beta / 3}$ (see Daly 1994, and Guerra and Daly 1995, 1996). The data may be combined in two independent ways to estimate $\beta$ and both indicate $\beta \simeq 2 \pm 0.5$, and this result is independent of the cosmological model assumed. The physical reason for the anticorrelation is related to the energy extraction mechanism. The data suggest that $L_{j} \propto E_{i}^{3}$ for $\beta \simeq 2$, which in turn may be used to constrain models for the energy extraction.

De Young: If the radio source lifetime decreases with redshift, then the number of sources per unit comoving volume should decrease with increasing redshift. Have you looked for this?

Daly: Not as yet, but this would be a very interesting effect to look for, and could be used to infer something about the evolution of the underlying radio luminosity function for these sources.

Wilson: Do you think that the decline of the linear sizes of the radio sources toward higher redshift is related to the higher inverse Compton losses of the relativistic electrons on the microwave background (the energy density of which increases with redshift)? This effect would tend to "snuff out" large, old radio sources at higher redshift.

Daly: It is true that inverse Compton losses become significant at high redshift as pointed out by Rees and Setti (1968, Nature, 219, No. 5150, 127). However, it is now known that the youngest relativistic electrons 
are near the radio hotspot, and the older electrons, which would be effected by the inverse Compton cooling, are closer to the center of the source. The effect you mention may make it difficult to observe the radio bridges of high redshift radio sources, but it is unlikely to effect the radio emission near the hotspots, which produce most of the radio emission and are used to define the source size. Thus, we must look to some other physical effect that would cause higher redshift sources to be smaller; we think they are smaller because they have higher beam powers and shorter lifetimes, leading to smaller sources.

Gopal-Krishna: If at higher redshifts radio source engines extinguish faster (followed by a decay in radio luminosity), then wouldn't one expect the radio luminosity function to get increasingly weighted in favor of lower luminosity radio galaxies at earlier cosmological epochs?

Daly: This would be the case if we were able to detect sources with low beam power. It turns out that the beam power and radio power are proportional to one another (see Wan and Daly 1996c). Because the sources studied come from flux limited samples, the radio power increases with redshift. We also see that the beam power increases with redshift, and deduce that this arises because of the correlation between radio power and beam power. Thus, at high redshift we only observe the most powerful sources. In this sense, the decrease of the source size with redshift is a selection effect caused by the fact that at high redshift we observe sources with higher beam power, these sources have shorter lifetimes, and thus are smaller. However, there is another parameter that enters into the source size, which is the lobe propagation velocity. Thus, the decrease of the source sizes with redshift is much weaker than the increase of the beam power with redshift; this follows because the lobe propagation velocity increases with redshift, probably because it depends on the beam power.

Discussion of the paper presented by $\underline{L A C Y}$ (p. 321)

De Young: In the $z=4.41$ object, what is the limiting magnitude in the $\mathrm{K}$ band image?

Lacy: $\mathrm{K}>21.5$

(Since the conference ended we have achieved a marginal detection at $\mathrm{K} \simeq 22$ ).

Schilizzi: How strong is the radio emission in $6 \mathrm{C} 0140+326$ ? 
Lacy: The radio flux density at $151 \mathrm{MHz}$ is $1.0 \mathrm{Jy}$.

Discussion of the paper presented by FERETTI (p. 333)

Stocke: Could you please locate the Coma "relic" radio source on the X-ray map of the Coma Cluster.

Feretti: The relic $1253+275$ lies beyond the sub-cluster centered on NGC 4839 , in the same direction.

Partridge: What is the typical age of the relic sources in clusters?

Feretti: It is of the order of $10^{8}$ yrs.

Meisenheimer: Which value of magnetic field did you use for the age estimate and how does that compare with the energy density in the cosmic microwave background?

Feretti: The age of Coma $C$ using the equipartition magnetic field is $10^{8}$ yr. Using the magnetic field derived from rotation measure, the age is much shorter. In the latter case, the energy density of the magnetic field is larger than that in the microwave background, whose equivalent magnetic field is $\sim 4 \mu G$.

Dogiel: Where does the energy of relativistic electrons in the Coma cluster halo come from? Is it a part of kinetic energy of the tail galaxy orbiting around the cluster center or is it the energy of CR electrons emitted by this galaxy?

Feretti: The relativistic electrons are likely to be deposited by the tailed radio galaxy NGC4869, and reaccelerated by the energy available from a cluster merger process and possibly galactic wakes.

Eilek: The problem of sources of the relativistic electrons is important; they cannot diffuse from the cluster center fast enough to supply the haloes. Do you know if all six of the large-scale haloes have outlying radio galaxies which can supply the particles?

Feretti: The best studied haloes, ie. those in A1656, A2255, A2256 and A2319, have embedded tailed radio galaxies.

The clusters A2163 and A2218 are distant, and less studied. Higher resolution data are necessary to distinguish discrete sources from the broad halo radio emission. 
Harris: We expect little or no polarization for haloes because of turbulence making smaller cell sizes and mixing in thermal plasma. Could the quasilinear polarized structure in A2256 (20\% polarization) be the remnant of an old tailed radio galaxy?

Feretti: The halo in A2256 is complex: it actually consists of a very low brightness region at the cluster center and a stronger, polarised feature in the $\mathrm{N}-\mathrm{W}$ part. This higher brightness region could be the remnant of more tailed radio galaxies.

\section{Discussion of the paper presented by BÖHRINGER (p. 357)}

Binette: Cooling shocks due to the radio jets might explain the excess emission in X-ray emission related to the radio-structure in M87. Why are shocks not considered any more as a mechanism which compresses the gas and adjusts pressure imbalances?

Böhringer: The main problem is not a problem of pressure imbalance, because we see that the gas is colder in the radio lobes than outside and, with the higher density inferred from the high surface brightness, the lobe gas and the surrounding ICM can still be at pressure equilibrium. The main question is: Why is the gas cooler in the lobes instead of being heated by the disturbances? It may thus be possible that adiabatic waves or weak shocks introduce a temporary clumpiness in the gas speeding up the radiative cooling rather than heating the ICM.

Fraix-Burnet: What is the meaning of the dark blue region West of the nucleus on the M87 X-ray map?

Böhringer: The dark blue region in the X-ray halo image of M87 is a surface brightness deficiency located roughly at the same position angle as the optical and radio jet. Therefore it could in principle also originate from an X-ray gas displacement as in the case of NGC 1275. But this feature is not so significant as in NGC 1275.

Macchetto: I do not understand how you get baryonic densities as high as $30 \%$ in clusters such as A2218. Work by Ellis et al, using HST data and ground based redshifts, seem to indicate $M / L \approx 100$ and upper limits to the baryonic density of $10 \%$.

Böhringer: The examples I have shown refer to nearby clusters and a Hubble constant of 50 . A baryon mass fraction of $30 \%$ is derived if we integrate over the whole cluster out to $3 \mathrm{Mpc}$ (in the case of Coma or Perseus). If one looks only at the central part of the cluster and uses 
$H_{0}=100 \mathrm{kms}^{-1} \mathrm{Mpc}^{-1}$ the baryon fraction is lower. The lensing results by Ellis et al may refer to this case. The discrepancy for $M / L$ is more difficult to understand; for $H_{0}=50$ we usually find values from 100-300. for $H_{0}=100$ the values are higher by a factor of two.

Discussion of the paper presented by YAMASHITA (p. 363)

Simkin: How does the temperature asymmetry which you found in the Coma cluster compare with the radio galaxy distribution?

Yamashita: We would like to compare the ASCA result with the radio galaxy distribution. We have not checked any radio data in the Coma region. If the high temperature region coincides to dense distribution of radio galaxies, it seems that the contribution of non-thermal component is expected to be significant. 